\title{
Porcine epidemic diarrhea virus nsp14 inhibits NF-KB pathway activation by targeting the IKK complex and p65
}

\author{
Shasha Li ${ }^{\dagger}$, Fan Yang ${ }^{\dagger}$, Caina Ma, Weijun Cao, Jinping Yang, Zhenxiang Zhao, Hong Tian, Zixiang Zhu* and
} Haixue Zheng*

\begin{abstract}
Coronaviruses (CoVs) are a group of related enveloped RNA viruses that have severe consequences in a wide variety of animals by causing respiratory, enteric or systemic diseases. Porcine epidemic diarrhea virus (PEDV) is an economically important CoV distributed worldwide that causes diarrhea in pigs. nsp14 is a nonstructural protein of PEDV that is involved in regulation of innate immunity and viral replication. However, the function and mechanism by which nsp14 modulates and manipulates host immune responses remain largely unknown. Here, we report that PEDV nsp14 is an NF-KB pathway antagonist. Overexpression PEDV nsp14 protein remarkably decreases SeV-, poly (I: C)- and TNF-a-induced NF-KB activation. Meanwhile, expression of proinflammatory cytokines is suppressed by nsp14. nsp14 inhibits the phosphorylation of IKKs by interacting with IKKs and p65. Furthermore, nsp14 suppresses TNF-a-induced phosphorylation and nuclear import of p65. Overexpression nsp14 considerably increases PEDV replication. These results suggest a novel mechanism employed by PEDV to suppress the host antiviral response, providing insights that can guide the development of antivirals against CoVs.
\end{abstract}

Keywords: CoVs, PEDV, nsp14, NF-kB, innate immunity

\section{Introduction}

Coronaviruses (CoVs) are a group of related enveloped RNA viruses within the family of Coronaviridae in the order of Nidovirales that consist of four genera, Alphacoronavirus, Betacoronavirus, Deltacoronavirus and Gammacoronavirus (Gorbalenya et al. 2004; Woo et al. 2012). CoVs have severe health consequences by causing respiratory, enteric or systemic diseases in various animals. Some CoVs are lethal to their hosts, such as the $\mathrm{CoVs}$ that cause severe acute respiratory syndrome (SARS), Middle-east respiratory syndrome (MERS) and

\footnotetext{
* Correspondence: zhuzixiang@caas.cn; haixuezheng@163.com

'Shasha Li and Fan Yang contributed equally to this work.

State Key Laboratory of Veterinary Etiological Biology, National Foot and Mouth Diseases Reference Laboratory, Key Laboratory of Animal Virology of Ministry of Agriculture, Lanzhou Veterinary Research Institute, Chinese Academy of Agricultural Sciences, Lanzhou, Gansu 730046, China
}

COVID-19 in humans. Certain CoVs, including infectious bronchitis virus (IBV), porcine epidemic diarrhea virus (PEDV), and ferret systemic coronavirus (FSC), are lethal to animals (Haake et al. 2020).

CoVs have positive single-stranded RNA viral genomes ranging from 25 to $32 \mathrm{~kb}$, which encode a series of structural, accessory and nonstructural proteins. Structural proteins conclude nucleocapsid $(\mathrm{N})$, membrane $(\mathrm{M})$, spike (S), and envelope (E) proteins (de Artika et al. 2020), and ORF3 encodes a hypothetical accessory protein. Two large open reading frames (ORFs), ORF1a and ORF1b, compose of major part of the viral genome and encode two large replicase polyproteins (ppla and pplab), which are subsequently cleaved by viral proteases into 16 nonstructural proteins (nsps) (Ziebuhr et al. 2000). These nsps, together with other viral proteins and cellular factors, assemble into a large replication-

(c) The Author(s). 2021 Open Access This article is licensed under a Creative Commons Attribution 4.0 International License, which permits use, sharing, adaptation, distribution and reproduction in any medium or format, as long as you give appropriate credit to the original author(s) and the source, provide a link to the Creative Commons licence, and indicate if changes were made. The images or other third party material in this article are included in the article's Creative Commons licence, unless indicated otherwise in a credit line to the material. If material is not included in the article's Creative Commons licence and your intended use is not permitted by statutory regulation or exceeds the permitted use, you will need to obtain permission directly from the copyright holder. To view a copy of this licence, visit http://creativecommons.org/licenses/by/4.0/ The Creative Commons Public Domain Dedication waiver (http://creativecommons.org/publicdomain/zero/1.0/) applies to the data made available in this article, unless otherwise stated in a credit line to the data. 
transcription complex (RTC). RTCs are associated with double membrane vesicles derived from the endoplasmic reticulum and are responsible for viral RNA replication and transcription of subgenomic RNAs.

The innate immune response is critical for defending the host from various invading pathogens. Viral pathogen-associated molecular patterns (PAMPs) are recognized by pattern recognition receptors (PRRs), which induce the production of inflammatory cytokines and type I interferons (IFNs) by activating transcription factor nuclear factor kappa $\mathrm{B}(\mathrm{NF}-\mathrm{kB})$ and IFN regulatory factors. Activation of NF- $\mathrm{kB}$ signaling pathway is crucial for innate immunity and other processes involving cellular survival, proliferation and differentiation. NF- $\mathrm{kB}$ family consists of five members: p50, p52, p65, RelB and c-Rel (Hayden and Ghosh 2008). Classical NF$\kappa B$ signaling pathway activation requires the release of NF- $\mathrm{kB}$ p50/p65 dimers, while nonclassical NF- $\mathrm{kB}$ signaling pathway activation requires the formation of $\mathrm{p} 52 / \mathrm{Rel}$ $\mathrm{B}$ dimers. In classical NF- $\mathrm{KB}$ signaling pathway, p65/p50 dimers are sequestered in cytoplasm through interaction

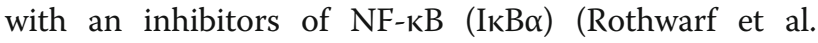
1998). Upon viral infection, ІкB $\alpha$ is phosphorylated by I $\mathrm{B}$ kinase (IKK $\alpha$ and IKK $\beta$ ) complex and degraded in proteasome, thereby releasing p65/p50 dimers for phosphorylation and translocation into nucleus (Kanarek and Ben-Neriah 2012; Liu et al. 2012). The major upstream receptors mediating NF- $\mathrm{KB}$ activation include toll-like receptors (TLRs), retinoic acid-inducible gene I (RIG-I), tumor necrosis factor receptor (TNFR), and interleukin 1 receptor type 1 (IL-1R1). The downstream proteins regulated by these receptors mainly include myeloid differentiation primary response gene 88 (MyD88), Toll/IL1 receptor (TIR)-containing adaptor-inducing IFN- $\beta$ (TRIF), and mitochondrial antiviral signaling protein (MAVS).

To establish successful infection, various CoVs have evolved multiple strategies to evade the host antiviral response. During CoV infection, several replicase proteins functioned as interferon antagonists to block the expression of host antiviral proteins. In addition, CoV nsp14 and nsp16 exhibit N7-methyltransferase (N7-MTase) and 2'O-methyltransferase activities, respectively, which catalyze the formation of a 5'cap-1 structure, preventing recognition of viral RNA by PRRs (Chen et al. 2009; Decroly et al. 2008). All CoV nsp14s have 3 '-to-5' exoribonuclease (ExoN) activity and N7-methyltransferase activity (N7-MTase) (Chen et al. 2009; Minskaia et al. 2006). N7-MTase activity is critical for translation of the viral genome and prevents the sense of viral mRNAs as a "nonself" signature by host PRRs (Becares et al. 2016). ExoN activity is critical for the fidelity of viral replication (Minskaia et al. 2006). Previous studies have suggested that $\mathrm{CoV}$ nsp14 plaied potential roles in modulation of innate immunity (Becares et al. 2016; Case et al. 2017). Mutation of N7-MTase domain of murine hepatitis virus (MHV) nsp14 enhances its sensitivity to the host innate immune response, and ExoN activity of nsp14 is essential for its resistance to the antiviral innate immune response (Case et al. 2017). A recent study showed that N7 MTase-deficient PEDV was defective in replication, but infection with this virus resulted in increased secretion of type I and III IFNs (Lu et al. 2020). However, the role and regulatory mechanisms of PEDV nsp14 in innate immunity are still poorly understood.

PEDV is an alphacoronavirus that causes acute and highly contagious enteric viral disease in pigs. Starting in 2010, a significant increase in PEDV outbreaks occurred in the USA and Asia countries, giving rise to severe economic consequences worldwide (Sun et al. 2012; Tian et al. 2014). Variants of highly virulent PEDV strains that have contributed to the occurrence of these outbreaks were identified. The immune evasion of these PEDV strains is closely associated with severe clinical pathogenesis. Several PEDV proteins have been reported to interrupt RIG-I signaling pathway to inhibit IFN- $\beta$ production.

Although RIG-I signaling pathways regulated by PEDV viral proteins have been extensively characterized, how PEDV regulates proinflammatory cytokine expression remains largely unknown. Here, in this study, we identified PEDV nsp14 as an NF- $\mathrm{kB}$ antagonist. This research showed that nsp14 suppressed NF- $\mathrm{kB}$ signaling by interacting with $I K K \alpha / \beta$ and $p 65$ as well as interfering with the phosphorylation of $I K K \alpha / \beta$ and IKB $\alpha$, which subsequently blocked the degradation of IкB $\alpha$ and thus suppressed TNF- $\alpha$-induced p65 phosphorylation and nuclear translocation. Overall, our data identified a new NF- $\mathrm{KB}$ pathway antagonist of PEDV and a novel antagonistic mechanism evolved by PEDV to inhibit proinflammatory cytokine expression.

\section{Results \\ PEDV nsp14 inhibits SeV infection-induced NF-KB promoter activation}

PEDV has the ability to modulate host immune and inflammatory responses (Cao et al. 2015; Zhang et al. 2017a, 2017b). PEDV infection significantly inhibits NF$\kappa B$ activation and the production of proinflammatory cytokines in porcine epithelial cells (Zhang et al. 2017a, 2017b). nsp14 is a potential viral factor that blocks NF$\mathrm{kB}$ activation; however, the mechanism remains unknown (Zhang et al. 2017a, 2017b). To evaluate the effect of PEDV nsp14 on NF-kB activation, a dual luciferase reporter assay was performed to determine the antagonistic effect of nsp14 on NF- $\mathrm{kB}$ promoter activation induced by Sendai virus (SeV). PEDV nsp10 that has no effect on NF-kB activation (Zhang et al. 2017a, 
2017b), was used as a control. Expression of PEDV nsp10 and nsp14 was confirmed by Western blotting (Fig. 1A). The activation of NF- $\mathrm{KB}$ promoter induced by $\mathrm{SeV}$ was significantly inhibited by PEDV nsp14 but not by nsp10 (Fig. 1A). In addition, inhibitory effect of nsp14 on NF- $\mathrm{kB}$ activation was dose dependent (Fig. 1B). Overexpression nsp14 suppressed activation of NF- $\mathrm{kB}$ promoter in porcine intestinal epithelial cells (IPEC-J2 cell line) (Fig. 1C). These data suggest that nsp14 is an important antagonistic factor of PEDV to inhibit $N F-\kappa B$ activation.

\section{PEDV nsp14 blocks the expression of NF-KB-mediated proinflammatory cytokines}

As described above, PEDV nsp14 suppressed activation of NF- $\mathrm{kB}$ promoter. To determine if nsp14 inhibits the

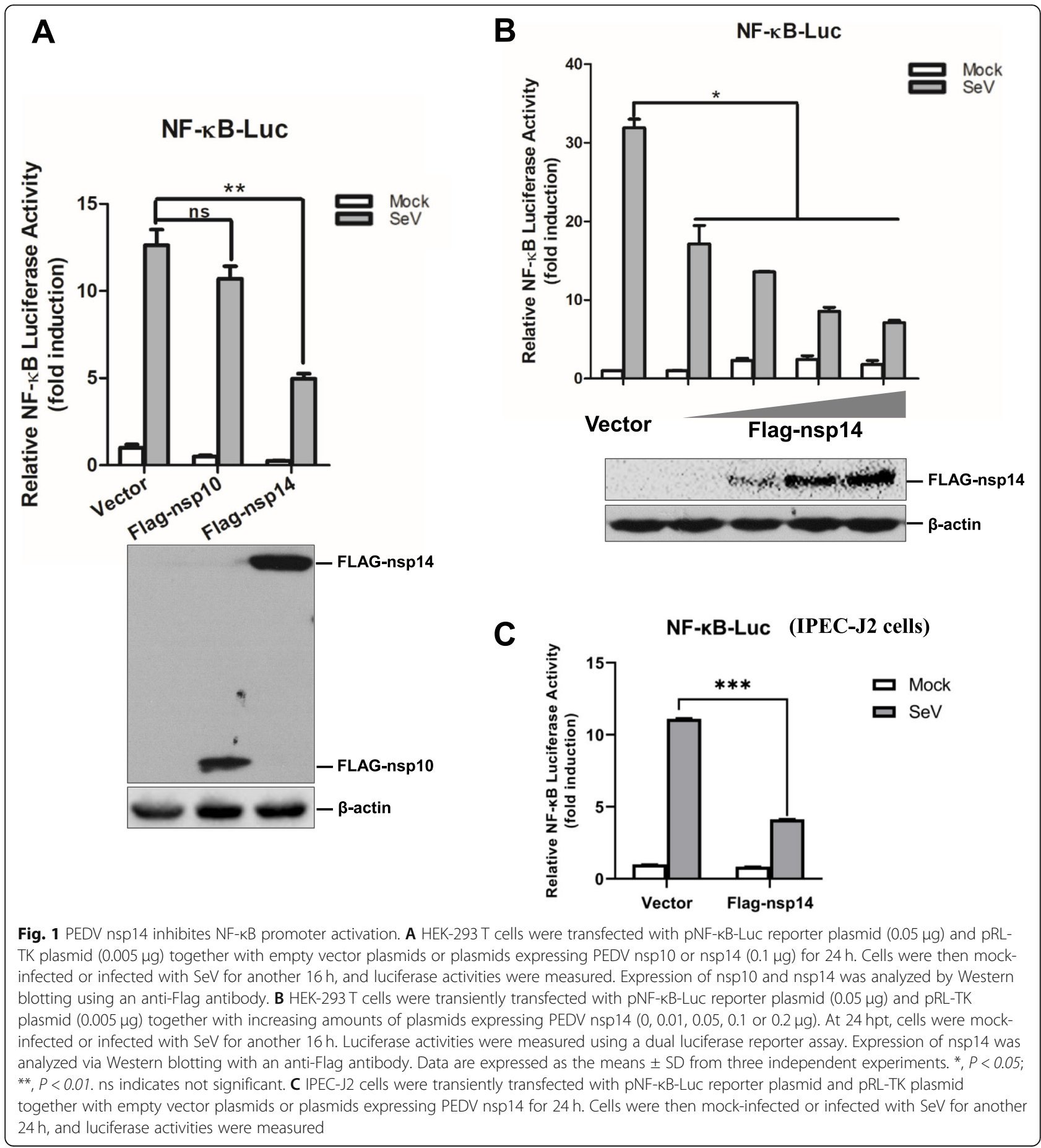



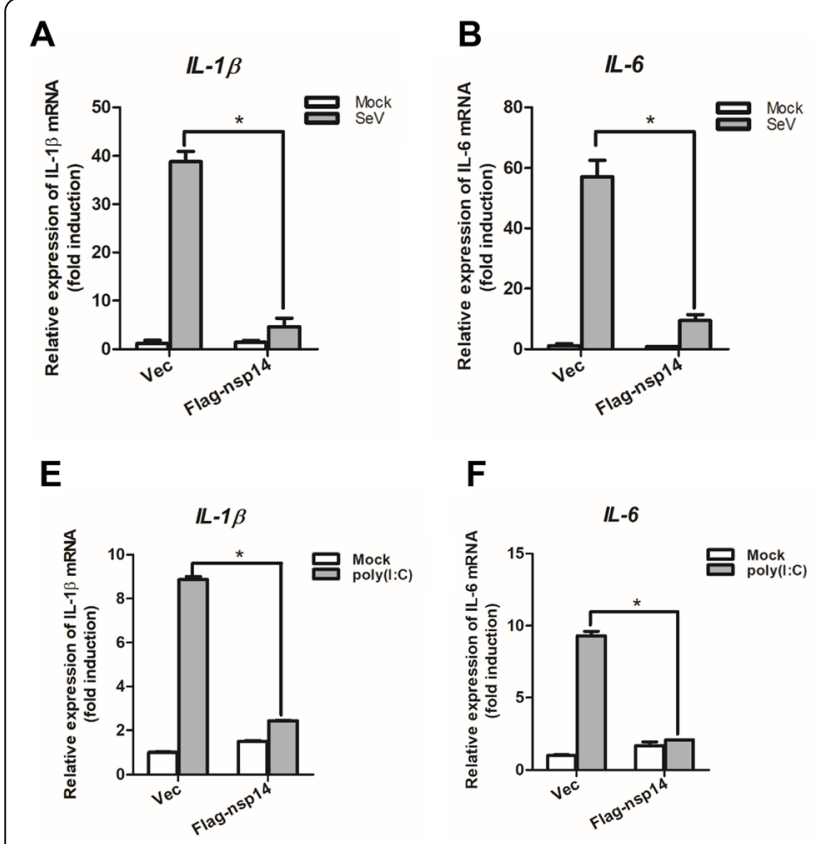

$\mathbf{F}$

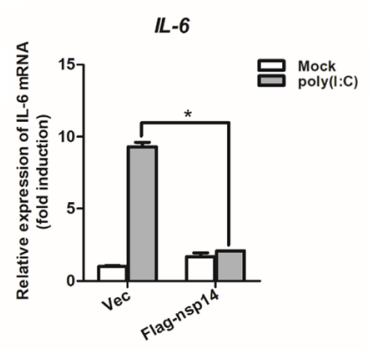

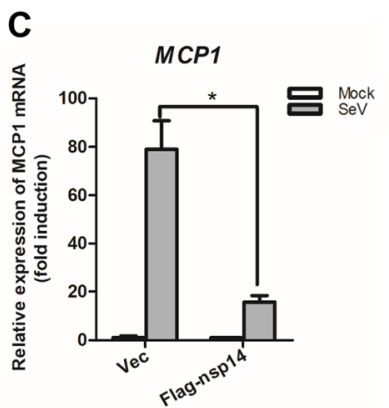

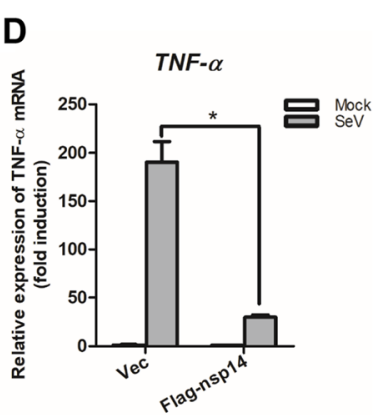

G

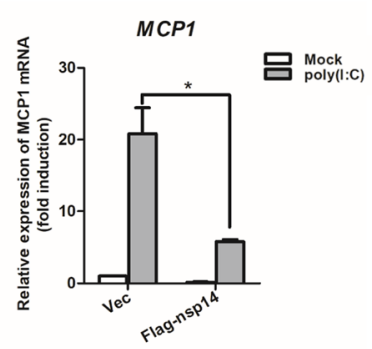

H

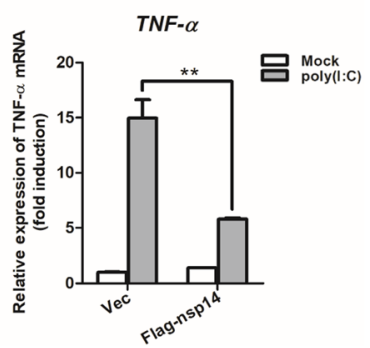

Fig. 2 PEDV nsp14 suppressed the expression of proinflammatory cytokines in HEK293T cells. A-D HEK293T cells were transfected with $1 \mu \mathrm{g}$ vector plasmids or Flag-nsp14 expression plasmids. At $24 \mathrm{hpt}$, cells were infected with SeV for $16 \mathrm{~h}$, andmRNA levels of IL-1 3 (A), IL-6 (B), MCP1 (C) and TNF-a (D) were measured via qPCR analysis. E-F HEK293T cells were transfected with $1 \mu \mathrm{g}$ vector plasmids or Flag-nsp14 expression plasmids. At $24 \mathrm{hpt}$, cells were transfected with $1 \mu \mathrm{g}$ poly (l:C) for $12 \mathrm{~h}$, and mRNA expression levels of IL-1 $\beta$ (E), IL-6 (F), MCP1 (G), and TNF-a (H) were measured via $\mathrm{qPCR}$ analysis. ${ }^{*}, P<0.05 ;{ }^{* *}, P<0.01$

expression of proinflammatory cytokines, HEK-293 T cells were transfected with p3xFLAG-CMV 7.1 vector plasmids or Flag-nsp14 expression plasmids for $24 \mathrm{~h}$ and then mock-infected or infected with $\mathrm{SeV}$ for $16 \mathrm{~h}$. SeV infection strongly induced the expression of IL-1 $\beta$, IL-6, MCP1 and TNF- $\alpha$ (Fig. 2A-D). However, expression of these proinflammatory cytokines was significantly inhibited in the presence of PEDV nsp14. These data indicate that PEDV nsp14 decreases the expression of proinflammatory cytokines induced by NF-kB. To further confirm the nsp14-mediated inhibitory effect on proinflammatory cytokine expression, HEK-293 T cells were transfected with poly (I:C) to activate NF- $\mathrm{kB}$ signaling pathway, and the expression of proinflammatory cytokines in the presence or absence of PEDV nsp14 was evaluated via qPCR. Poly (I:C) transfection remarkably induced the expression of IL-1 $\beta$, IL-6, MCP1 and TNF$\alpha$. However, expression of these proinflammatory cytokines was considerably suppressed by PEDV nsp14 (Fig. $2 \mathrm{E}-\mathrm{H})$. The regulatory effect of nsp14 on mRNA expression of IL- $1 \beta$, IL- 6 and TNF- $\alpha$ induced by SeV and TNF- $\alpha$ in IPEC-J2 cells was further evaluated, and the results showed that overexpression of nsp14 considerably inhibited the expression of these proinflammatory cytokines induced by both $\mathrm{SeV}$ (Fig. 3A-C) and TNF- $\alpha$ (Fig. 3D-F) in porcine intestinal epithelial cells. These data confirm that PEDV nsp14 blocks the expression of pro-inflammatory cytokines mediated by NF-kB.

\section{PEDV nsp14 does not downregulate the protein levels of various}

NF-KB signaling pathway components

Activation of RIG-I-mediated NF- $\mathrm{B}$ pathway requires many components, including RIG-I, VISA, IKK $\alpha, \mathrm{IKK} \beta$, $\mathrm{I} \kappa \mathrm{B} \alpha$ and $\mathrm{p} 65$. Protein abundance of these signaling components directly affects activation of NF- $\kappa B$ pathway. To investigate the component that may potentially be targeted by PEDV nsp14, effect of nsp14 on the expression of RIG-I, VISA, IKK $\alpha, I K K \beta$, I $\mathrm{B} \alpha$ and p65 were investigated. HEK-293 T cells were cotransfected with plasmids expressing Flag-nsp14 or vector plasmids along with plasmids encoding HA-tagged adaptor molecules (RIG-I, VISA, IKK $\alpha$, IKK $\beta$, I $\mathrm{B} \alpha$ or p65). Protein abundance of these molecules was evaluated via Western blotting analysis. As in Fig. 4, PEDV nsp14 did not affect protein expression of various components of NF- $\mathrm{kB}$ signaling pathway.

\section{PEDV nsp 14 interactes with IKKa, IKK $\beta$ and $p 65$}

PEDV nsp14 does not affect protein expression of the components of NF- $\mathrm{kB}$ signaling pathway. We further evaluated whether nsp14 interacts with any of these 


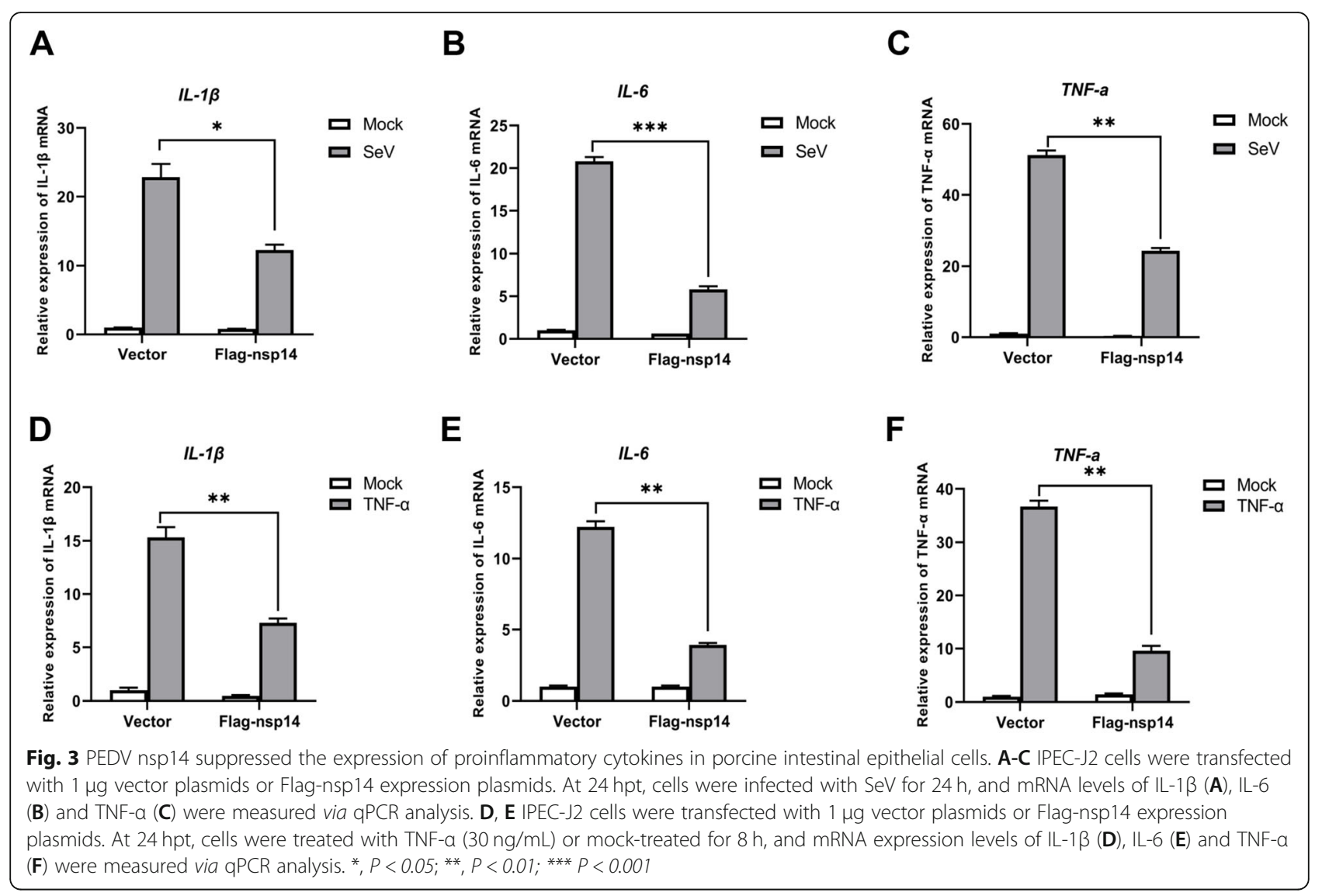

components. The interaction status between PEDV nsp14 and these components was investigated using CoIP assays. HEK-293 T cells were transfected with vector plasmids or Flag-nsp14 expression plasmids and with plasmids expressing HA-tagged RIG-I, VISA, IKK $\alpha$, $\mathrm{IKK} \beta, \mathrm{I} \mathrm{K} B \alpha$ or $\mathrm{p} 65$. The lysates were pulled down with anti-Flag or IgG antibody and subjected to Western blotting analysis using the indicated antibodies. As

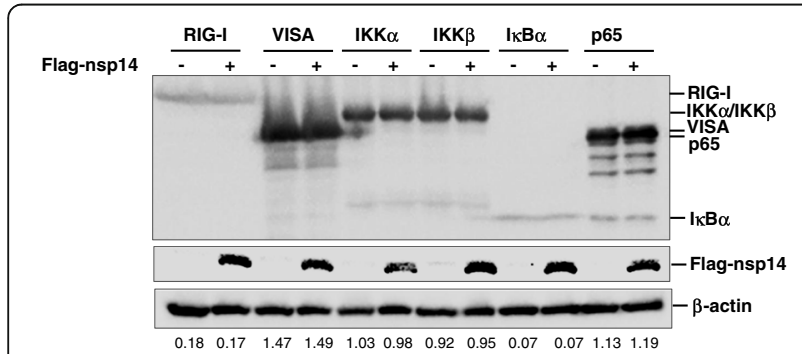

Fig. 4 PEDV nsp14 did not inhibit protein expression of various components of NF-KB pathway. HEK293T cells were transfected with constructs expressing HA-tagged RIG-I, VISA, IKKa, IKKß, IKBa or p65 along with vector plasmids or Flag-nsp10 expression plasmids. At 24 hpt, cell lysates were prepared for Western blotting analysis with the indicated antibodies. The relative abundance of the detected proteins was determined by densitometric analysis using ImageJ software shown in Fig. 5A, IKK $\alpha$, IKK $\beta$ and p65 were pulled down by nsp14. A reverse Co-IP experiment was further performed to confirm the interaction. Similarly, nsp14 was efficiently pulled down by IKK $\alpha$, IKK $\beta$ and p65 (Fig. $5 \mathrm{~B})$. Interaction between nsp14 and IKK $\alpha, \mathrm{IKK} \beta$ or p65 was also evaluated in porcine IPEC-J2 cells and got the same conclusion (Fig. 5C). These data indicate that nsp14 interacts with IKK $\alpha$, IKK $\beta$ and p65.

\section{PEDV nsp 14 inhibites IKKa/ $\beta$ phosphorylation and decreased NF-KB nuclear translocation}

TNF- $\alpha$ is an effective agonist of NF- $\kappa B$ signaling pathway. TNF- $\alpha$ treatment results in activation of downstream IKKs (Li et al. 2009). IКB $\alpha$ is phosphorylated by the activated IKKs, resulting in IкB $\alpha$ ubiquitination and degradation, and then, p65/p50 dimer is released from $\mathrm{I \kappa} B \alpha$ and translocated into nucleus (Chan et al. 2000; Pires et al. 2018; Rahman and McFadden 2011). To gain insights into the molecular mechanisms by which nsp14 suppresses NF- $\mathrm{kB}$ activation, effect of nsp14 on IKK $\alpha / \beta$, IKB $\alpha$ and p65 phosphorylation was examined. HEK-293 T cells were transfected with vector plasmids or Flag-nsp14 expression plasmids. At $24 \mathrm{~h}$ post transfection (hpt), cells were treated with TNF- $\alpha$ for $30 \mathrm{~min}$, and then, 

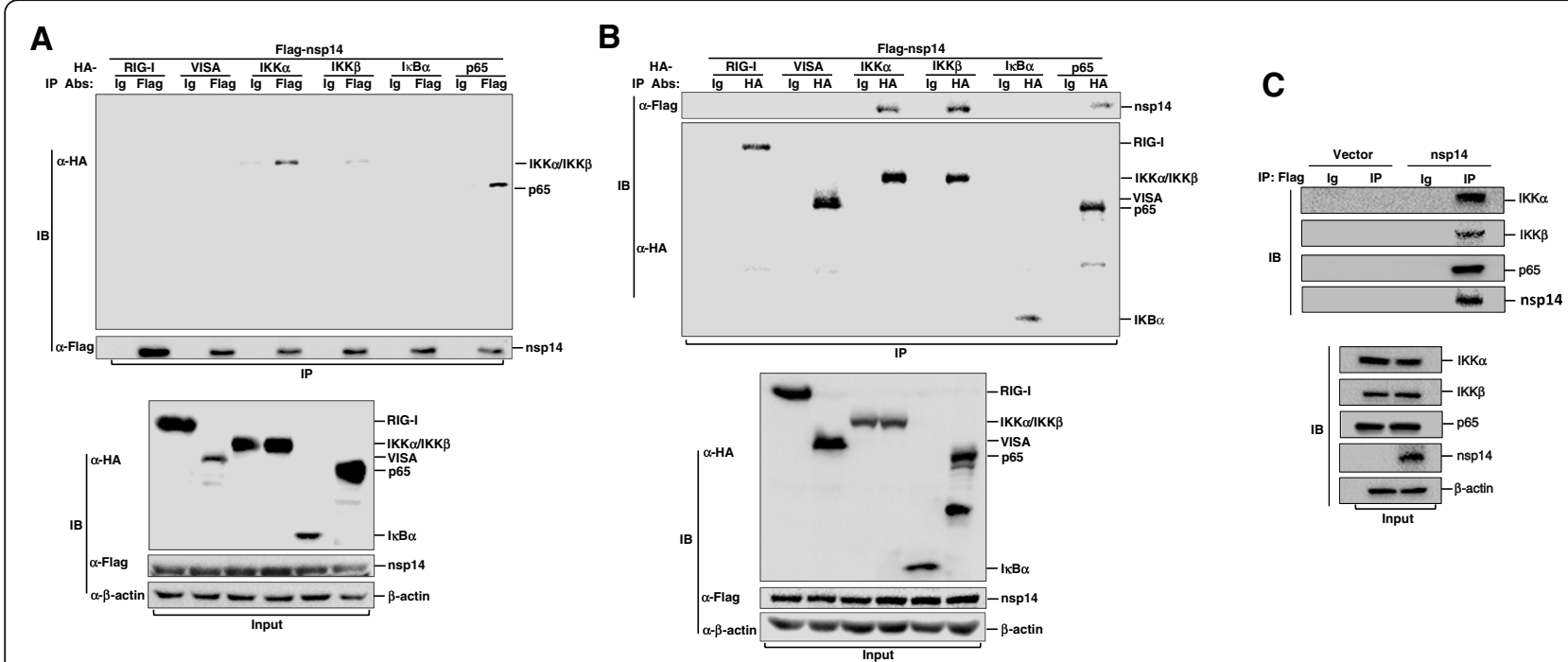

Fig. 5 PEDV nsp14 interactes with IKKa, IKKß and p65 in NF-KB pathway. A and $\mathbf{B}$ HEK-293 T cells were cotransfected with Flag-nsp14 expression plasmids and the indicated HA-tagged molecule expression plasmids (RIG-I, VISA, IKKa, IKKß, IKBa or p65). At 36 hpt, cell lysates were prepared for Co-IP experiments using anti-Flag antibody (A), anti-HA antibody or (B) nonspecific mouse lgG. Western blotting analysis was carried out with the indicated antibodies to evaluate the whole cell lysates (WCLs) and immunoprecipitation complexes. C IPEC-J2 cells were transfected with Flagnsp14 expression plasmids for $36 \mathrm{~h}$. Then, cell lysates were prepared for Co-IP experiments using anti-Flag antibody or nonspecific mouse IgG. Western blotting analysis was carried out with the indicated antibodies to evaluate WCLs and IP complexes

phosphorylation levels of $I K K \alpha / \beta$, I $\mathrm{B} \alpha \alpha$ and $\mathrm{p} 65$ were assessed via Western blotting. As shown in Fig. 6A, TNF- $\alpha$ stimulation led to a significant increase in $\mathrm{IKK} \alpha / \beta, \mathrm{I} \kappa \mathrm{B} \alpha$ and $\mathrm{p} 65$ phosphorylation, but overexpression PEDV nsp14 remarkably inhibited this process. Meanwhile, TNF- $\alpha$ treatment downregulated

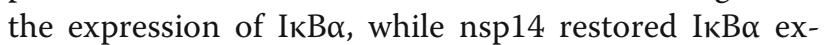
pression in TNF- $\alpha$-stimulated cells (Fig. 6A). These results indicate that nsp14 restrains phosphorylation of IKK complex, which in turn blocks NF-kB activation.

Nuclear translocation of p65 is an indicator of NF- $\mathrm{kB}$ activation. Subsequently, nuclear translocation of p65 in response to TNF- $\alpha$ treatment in absence and presence of nsp14 were assessed using an indirect immunofluorescence assay. TNF- $\alpha$ treatment caused increased nuclear translocation of p65. However, overexpression PEDV nsp14 decreased TNF- $\alpha$-induced nuclear translocation of p65 (Fig. 6B). Meanwhile, colocalization between nsp14 and p65 was also observed. These results suggest that PEDV nsp14 inhibits NF- $\mathrm{kB}$ nuclear translocation and contributes to the suppression of NF- $\mathrm{kB}$ pathway activation.

\section{Overexpression nsp14 protein enhances PEDV replication}

As a vital nonstructural protein, PEDV nsp14 plays important roles during viral infection. The results above showed that nsp14 suppressed NF-kB activaton and decreased early production of proinflammatory cytokines. To evaluate PEDV replication activity in cells overexpressing nsp14-, PEDV replication status in PK-15 cells was investigated after transfection with nsp14 expression plasmids. Cells were incubated with PEDV at an MOI of 1 at $24 \mathrm{hpt}$ and infected for $18 \mathrm{~h}$. Viral mRNA (Fig. 7A) and viral protein levels (Fig. 7B) were determined and used as an indicator of viral replication. Overexpression nsp14 considerably enhanced PEDV replication. Together, these data suggest that nsp14 plays an important role in PEDV replication.

\section{Discussion}

The most important function of NF- $\mathrm{kB}$ in the biological system is regulation of immune responses. It plays pivotal roles in immune homeostasis by regulating innate immune responses, inflammation, cell survival and cell proliferation (Poppe et al, 2017; Wullaert et al. 2011a, 2011b). Canonical NF- $\kappa B$ pathway is mediated mainly by PRRs and proinflammatory cytokine receptors, which induce degradation of the inhibitory factor IкB $\alpha$. The release of $\mathrm{I} \kappa \mathrm{B} \alpha$ from $\mathrm{NF}-\mathrm{\kappa B}$ complex contributes to nuclear translocation of NF- $\mathrm{kB}$, thus activates the expression of proinflammatory cytokines (Khatiwada et al. 2017; Rothwarf et al. 1998). Recognition of PAMPs by PRRs triggers host innate immune responses through activation of signaling cascades which eventually induce inflammatory responses and eradicate pathogens (Kumar et al. 2011; O'Neill and Bowie 2010). Hence, to counteract the host antiviral response, many viruses have evolved various strategies to evade NF- $\mathrm{kB}$ signaling (Smits et al, 2010). 


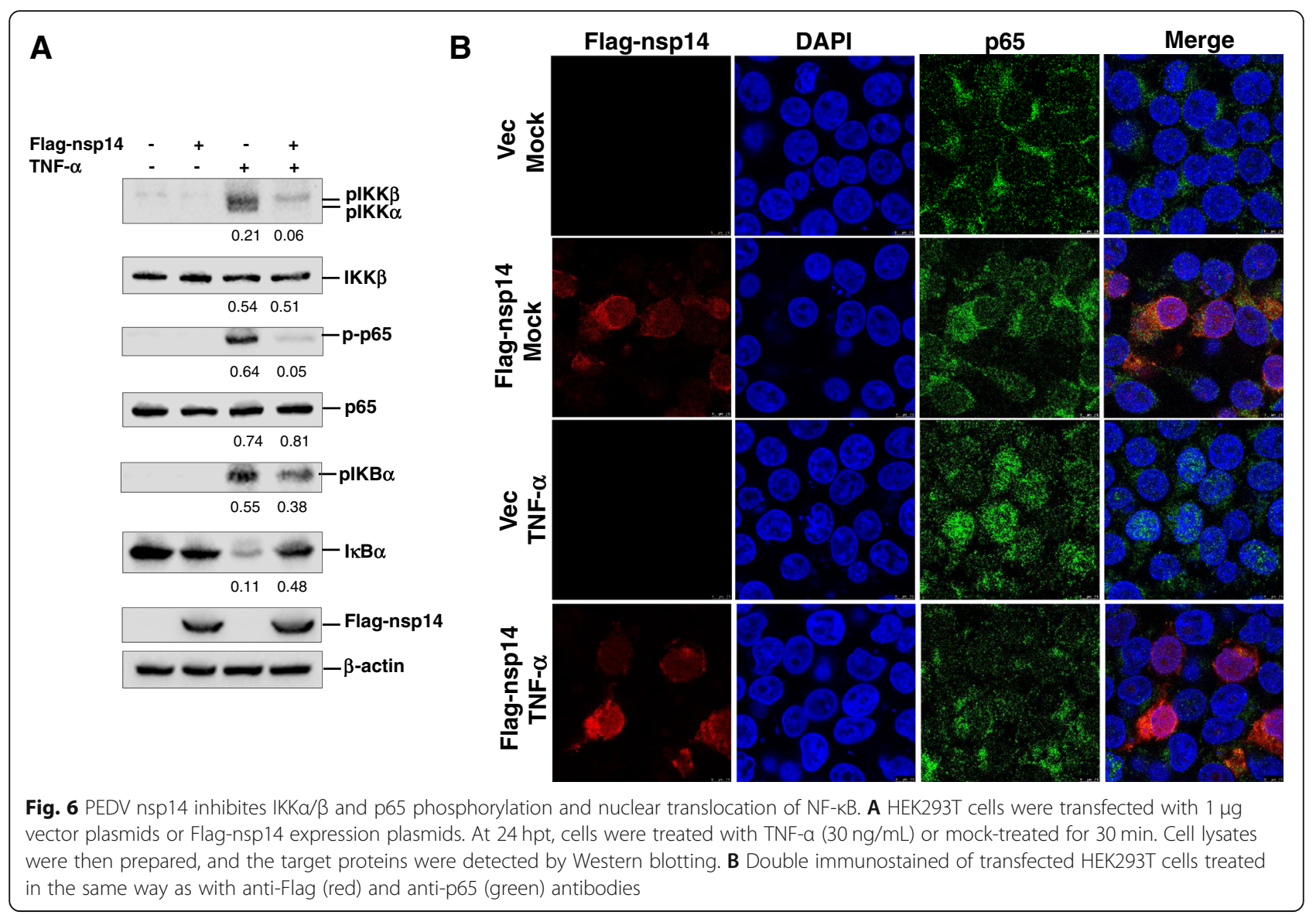

A

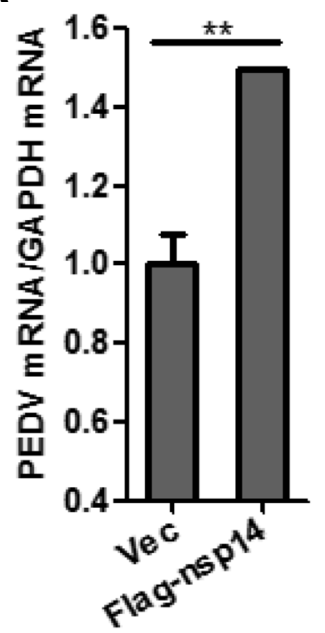

B

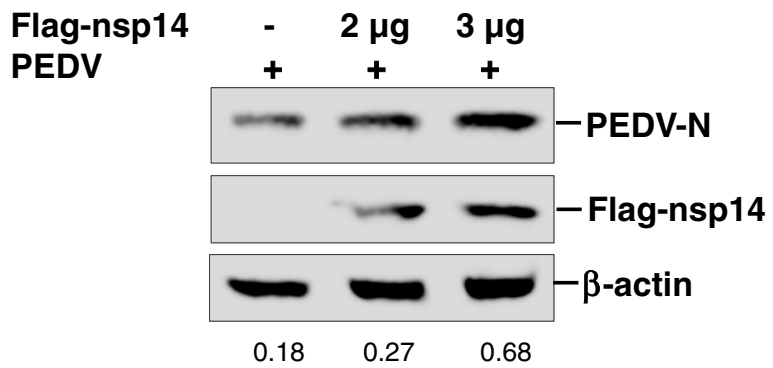

Fig. 7 PEDV nsp14 promotes PEDV replication. A PK-15 cells were transfected with $2 \mu \mathrm{g}$ vector plasmids or Flag-nsp14 expression plasmids. At 24 $\mathrm{hpt}$, cells were infected with PEDV at an MOI of 1 for $18 \mathrm{~h}$. The cells were then collected and subjected to qPCR analysis. B Western blotting analysis of transfected PK-15 cells using the indicated antibodies. The relative abundance of PEDV N protein was determined by densitometric analysis using ImageJ software 
Virion-associated protein 119 of virus inhibits NF- $\mathrm{KB}$ activation by interacting with NEMO, which finally results in a decrease in p65 nuclear translocation (Nagendraprabhu et al. 2017). Herpes simplex virus UL24, UL36, UL42, US3 and ICP0 proteins inhibit NF-kB activation by blocking the nuclear translocation of NF- $\mathrm{BB}$ or preventing the degradation of IKB $\alpha$ (Wang et al. 2014; Xu et al. 2017; Ye et al. 2017; Zhang et al. 2013a; Zhang et al. 2013b). MERS-CoV 4b protein interferes with NF$\kappa \mathrm{B}$-mediated innate immune response by binding karyopherin- $\alpha 4$ (KPNA4), thus preventing NF-kB nuclear translocation (Canton et al. 2018). In this study, we demonstrates that PEDV nsp14 interacts with $I K K \alpha / \beta$ and $\mathrm{p} 65$ to inhibit NF- $\mathrm{B}$ activation by inhibiting IKK $\alpha /$ $\beta$ phosphorylation and decreasing NF- $\mathrm{kB}$ nuclear translocation, thereby downregulating the transcription of various proinflammatory cytokines. As expected, overexpression PEDV nsp14 promoted viral replication. Thus, we deduced that PEDV nsp14-mediated suppressive effect on NF- $\mathrm{kB}$ activity is associated with PEDV-induced pathology and virulence in vivo.

All CoVs encode a bifunctional nsp14 protein containing 3'-to-5' exoribonuclease and N7-methyltransferase activities, which are essential for viral replication fidelity, mRNA stability, and translation (Chen et al. 2009; Minskaia et al. 2006). Function of SARS-CoV nsp14 has been widely studied (Chen et al. 2009; Chen et al. 2013; Jin et al. 2013; Ma et al. 2015). SARS-CoV is a betacoronavirus and thus differs from alphacoronavirus. A study on TGEV, a porcine alphacoronavirus, revealed that a recombinant virus with a mutation in the TGEV ExoN domain induced a reduced antiviral response compared to its parental virus (Becares et al. 2016). This suggested that porcine CoV nsp14 ExoN activity may be involved in regulation of innate immunity. In contrast, ExoN and N-7 MTase activity of betacoronavirus MHV play roles in suppression of the innate immune response (Case et al. 2016; Case et al. 2017). However, function of nsp14 proteins of other alphacoronaviruses remains largely unknown. A recent study characterized N-7 MTase of PEDV nsp14, which suggested that it is attenuated and triggers stronger production of type I and III IFNs ( $\mathrm{Lu}$ et al. 2020). These findings indicated that PEDV nsp14 N-7 MTase may be involved in the suppression of host innate immunity. Our data demonstrate that PEDV nsp14 inhibits NF-kB activity by targeting IKK complex and p65. nsp4 might block the formation of NF- $\mathrm{kB}$ complex and then inhibit the expression of various proinflammatory cytokines. These results support the hypothesis that PEDV nsp14 could be a target for the development of live attenuated vaccine candidates and antiviral therapeutics.

Relationship between PEDV infection and innate immunity has been reported previously. PEDV infection inhibits type I IFN production and early production of proinflammatory cytokines (Zhang et al. 2017a, 2017b; Zhang et al. 2016). PEDV $\mathrm{N}$ protein, nsp1, PLP2 and nsp5 are responsible for the suppression of type I IFN production via different mechanisms (Wang et al. 2016; Zhang et al., 2018; Zhang et al. 2017a, 2017b; Zhang et al. 2016; Zheng et al. 2008). PEDV nsp1 suppresses the phosphorylation of $I \kappa B \alpha$ and blocks I $\mathrm{I} B \alpha$ degradation, which in turn inhibits p65 activation, but PEDV nsp1 does not affect phosphorylation of IKK $\alpha / \beta$ complex or interacts with IKK $\alpha / \beta$ (Zhang et al. 2017a, 2017b). Here, we report that PEDV nsp14 interacts with $I K K \alpha / \beta$ and p65 to inhibit phosphorylation of $I K K \alpha / \beta$ complex, which in turn blocks nuclear translocation of $N F-\kappa B$. This result suggests that both PEDV nsp14 and nsp1 regulate NF- $\mathrm{kB}$ signaling via different mechanisms.

\section{Conclusions}

In conclusion, this work identified PEDV nsp14 as a new $\mathrm{NF}-\kappa \mathrm{B}$ antagonist Nsp14 suppresses NF- $\mathrm{B}$ activity and decreases early production of proinflammatory cytokines by interacting with $I K K \alpha / \beta$ and $p 65$, which inhibits the phosphorylation of $I K K \alpha / \beta$ and p 65 and nuclear translocation of NF-kB (Fig. 8).

\section{Methods \\ Cells}

HEK-293 T, PK-15 and IPEC-J2 cells were maintained at $37^{\circ} \mathrm{C}$ with $5 \% \mathrm{CO}_{2}$ in Dulbecco's modified Eagle's medium (DMEM, Gibco) supplemented with 10\% heatinactivated fetal bovine serum (ExCell, FSP500).

\section{Plasmid constructs}

cDNA fragments encoding PEDV nsp10 and nsp14 were amplified from PEDV and inserted into a p3xFLAGCMV 7.1 eukaryotic expression vector to generate Flagtagged nsp10- and nsp14 expression plasmids. All constructed plasmids were confirmed by DNA sequencing.

\section{RNA extraction and RT-qPCR}

Monolayer HEK-293 T cells seeded in 12-well plates were transfected with vector plasmids or Flag-nsp14 expression plasmids. At $24 \mathrm{hpt}$, cells were mock-infected or infected with $\mathrm{SeV}$ for $16 \mathrm{~h}$ and then washed with PBS to remove cell debris. Total RNA was prepared and used for cDNA synthesis as described previously (Zhang et al. 2017a, 2017b). cDNA was then employed for quantitative PCR (qPCR) using ChamQ ${ }^{\text {im }}$ Universal SYBR ${ }^{\oplus}$ qPCR reagents (Vazyme) and an ABI QuantStudio5 real-time PCR system. The qPCR primers are listed in Table 1. Data were normalized to GAPDH expression. The relative expression of mRNA was evaluated using the $2^{-\Delta \Delta C t}$ threshold method (Livak and Schmittgen 2001). 


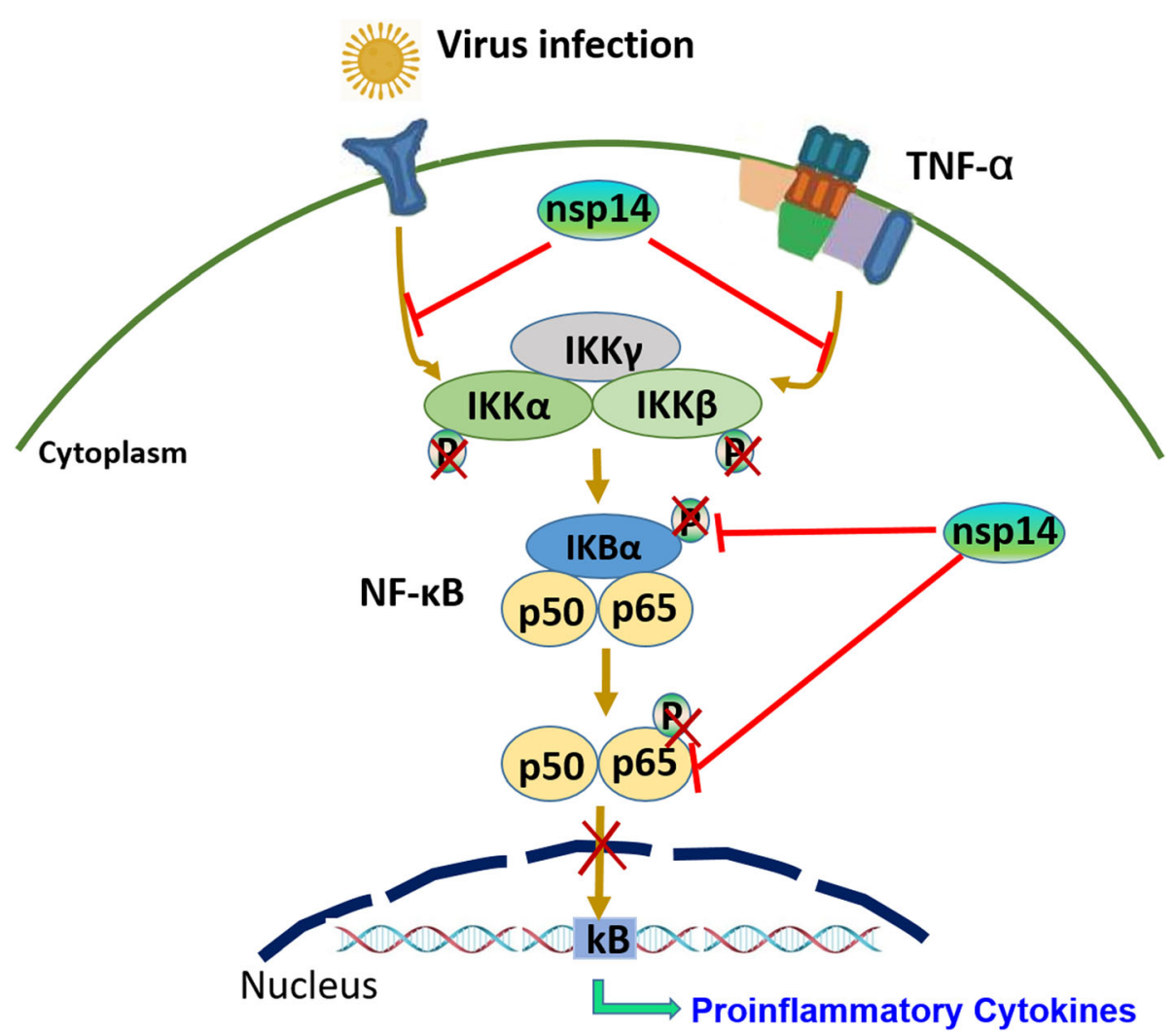

Fig. 8 Schematic diagram of PEDV nsp14 targets IKK complex and p65 to inhibit NF-KB activity. PEDV nsp14 negatively regulates NF-KB signaling pathway by interacting with $1 \mathrm{KKa} / \beta$ and $\mathrm{p} 65$, which inhibits the phosphorylation of $I \mathrm{KKa} / \mathrm{B}$ and $\mathrm{p} 65$, and nuclear translocation of NF-KB, leading to the decreased expression of early production of proinflammatory cytokines

\section{Antibodies and commercial cytokine}

Antibodies used for Western blotting analysis and immunofluorescence assays (IFAs) included anti-HA mouse antibody (Invitrogen), anti-Flag mouse antibody (Invitrogen), anti-human IKK $\beta$ rabbit antibody, anti$\mathrm{pIKK} \alpha / \mathrm{IKK} \beta$ rabbit antibody, anti-NF- $\mathrm{B}$ rabbit anti-

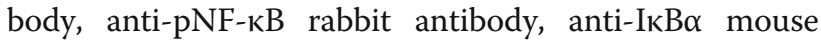
antibody, and anti-pIкB $\alpha$ rabbit antibody (Cell Signaling Technology). TNF- $\alpha$ (InvivoGen) was used to activate NF- $\kappa$ B pathway at a final concentration of $30 \mathrm{ng} / \mathrm{mL}$.

Table 1 qPCR primers used in the present study

\begin{tabular}{ll}
\hline Primers & \multicolumn{1}{c}{ Sequences } \\
\hline human-IL- $\beta-F$ & 5'- CAAAGGCGGCCAGGATATAA-3' \\
human-IL- $\beta-R$ & 5'- CTAGGGATTGAGTCCACATTCAG-3' \\
human-IL-6-F & 5'- TGACCCAACCACAAATGC-3' \\
human-IL-6-R & 5'- AGGAACTCCTTAAAGCTGCG-3' \\
human-MCP1-F & 5'- AGGTGACTGGGGCATTGATTG-3' \\
human-MCP1-R & 5'- GTCTCTGCCGCCCTTCTGTG-3' \\
human-TNF-F & 5'- AGAGGGAGAGAAGCAACTACA-3' \\
human-TNF-R & 5'- GGGTCAGTATGTGAGAGGAAGA-3' \\
\hline
\end{tabular}

\section{Western blotting}

HEK-293 T cells were cotransfected with vector plasmids or Flag-nsp14 along with HA-tagged adaptor molecule expression plasmids (RIG-I, VISA, IKK $\alpha$, IKK $\beta$, IкB $\alpha$ and p65) using Lipofectamine 2000. At $24 \mathrm{hpt}$, cells were lysed with NP-40 lysis buffer supplemented with complete protease inhibitors (Roche), and then, cell lysates were boiled at $95^{\circ} \mathrm{C}$ for $5 \mathrm{~min}$. The supernatant was then obtained by centrifugation. For detection of phosphorylated proteins, the transfected cells were treated with TNF- $\alpha$ for $30 \mathrm{~min}$ and lysed in the presence of an additional phosphatase inhibitor (Thermo Scientific Pierce). All samples were separated, transferred to nitrocellulose membranes (Millipore) and then incubated with the appropriate primary antibodies overnight at $4{ }^{\circ} \mathrm{C}$ after blocking. Appropriate HRP-conjugated secondary antibodies (Santa Cruz Biotechnology) were used to generate antigen-antibody complexes, which were visualized using ECL reagents (Advansta).

\section{Coimmunoprecipitation assay}

HEK-293 T cells were cotransfected with vector or Flagnsp14 along with HA-tagged adaptor molecule expression plasmids (RIG-I, VISA, IKK $\alpha, \mathrm{IKK} \beta$, IкB $\alpha$ and p65) 
using Lipofectamine 2000 for $36 \mathrm{~h}$. The cells were then lysed in lysis buffer supplemented with complete protease inhibitors (Roche). IPEC-J2 cells were transfected with vector or Flag-nsp14 using Lipofectamine 2000 for $36 \mathrm{~h}$. The cells were then lysed in lysis buffer supplemented with complete protease inhibitors (Roche). Cell lysates were incubated with $50 \mu \mathrm{L}$ protein $\mathrm{G}$ agarose beads and appropriate antibodies overnight at $4{ }^{\circ} \mathrm{C}$. After three washes of agarose beads, the immunoprecipitated complexes were subjected to Western blotting analysis.

\section{Immunofluorescence assay}

HEK-293 T cells were transfected with vector plasmids or Flag-nsp14 expression plasmids. At $24 \mathrm{hpt}$, cells were stimulated with TNF- $\alpha$ for $30 \mathrm{~min}$ and fixed with $4 \%$ paraformaldehyde. Cells were then permeabilized and incubated with appropriate antibodies as previously described (Nagendraprabhu et al. 2017). The nuclei were stained with DAPI (4'6'-diamidino-2-phenylindole) (Sigma) for $10 \mathrm{~min}$. The stained cells were analyzed using a Leica TCS SP5 II AOBS confocal microscope at the appropriate settings.

\section{Luciferase reporter assay}

HEK-293 T cells were cotransfected with HA- or Flagtagged protein expression plasmids, together with pNF$\kappa B$ luciferase reporter plasmid and Renilla internal control plasmids. IPEC-J2 cells were cotransfected with Flag vector or Flag-nsp14 and pNF-kB-Luc reporter plasmids as well as pRL-TK plasmids. The cell lysates were collected to measure the luciferase activity using a Dual Luciferase Assay System Kit (Promega) according to the manufacturer's instructions. The data indicate the relative firefly luciferase activity value normalized to the Renilla luciferase activity value.

\section{Statistical analysis}

Standard two-tailed unpaired Student's $t$ tests were employed to determine the significance of differences between groups. All results are presented as the means \pm standard deviation. A $P$-value less than 0.05 was considered statistically significant.

\footnotetext{
Acknowledgments

We sincerely thank Professor Hongbing Shu (Wuhan University, Wuhan, China) for kindly providing the luciferase reporter plasmids and various hemagglutinin (HA)-tagged plasmids and Professor Guangliang Liu for providing PEDV antibodies and valuable suggestions.
}

\section{Authors' contributions}

All authors have read and approved the final version of the manuscript. All authors have read and approved the final version of the manuscript. Designed the experiments: LSS, ZZX and ZHX; Performed the experiments: LSS, FY and MCN; Analyzed the data: LSS, CWJ and YJP; Provided reagents and material: ZZX and TH; Wrote the paper: LSS, ZZX and ZHX; Proofed the manuscript: $\mathrm{ZHX}$.

\section{Funding}

This work was supported by grants from the National Key R\&D Program of China (2018YFD0500103 and 2017YFD0501100), the Chinese Academy of Agricultural Science and Technology Innovation Project (CAAS-ASTIP-2021LVRI and Y2017JC55), and Central Public-interest Scientific Institution Basal Research Fund (1610312016013 and 1610312017003).

Availability of data and materials

Data will be shared upon request by the readers.

\section{Declarations}

Ethics approval and consent to participate Not applicable.

\section{Consent for publication}

Not applicable.

\section{Competing interests}

Author Haixue Zheng was not involved in the journal's review or decisions related to this manuscript. The authors declare no competing interests.

Received: 7 June 2021 Accepted: 14 September 2021

Published online: 14 October 2021

\section{References}

Becares, M., A. Pascual-Iglesias, A. Nogales, I. Sola, L. Enjuanes, and S. Zuñiga. 2016. Mutagenesis of coronavirus nsp14 reveals its potential role in modulation of the innate immune response. Journal of Virology 90 (11): 5399-5414. https://doi.org/10.1128/JVl.03259-15.

Canton, J., A.R. Fehr, R. Fernandez-Delgado, F.J. Gutierrez-Alvarez, M.T. SanchezAparicio, A. García-Sastre, S. Perlman, L. Enjuanes, and I. Sola. 2018. MERS-CoV $4 \mathrm{~b}$ protein interferes with the NF-KB-dependent innate immune response during infection. PLoS Pathogens 14 (1): e1006838. https://doi.org/10.1371/ journal.ppat.1006838.

Cao, L.Y., X.Y. Ge, Y. Gao, G. Herrler, Y.D. Ren, X.F. Ren, and G.X. Li. 2015. Porcine epidemic diarrhea virus inhibits dsRNA-induced interferon- $\beta$ production in porcine intestinal epithelial cells by blockade of the RIG-I-mediated pathway. Virology Journal 12 (1): 1-8. https://doi.org/10.1186/s12985-015-0345-x.

Case, J.B., A.W. Ashbrook, T.S. Dermody, and M.R. Denison. 2016. Mutagenesis of S-adenosyl-I-methionine-binding residues in coronavirus nsp14 N7methyltransferase demonstrates differing requirements for genome translation and resistance to innate immunity. Journal of Virology 90 (16): 7248-7256. https://doi.org/10.1128/JVI.00542-16.

Case, J.B., Y.Z. Li, R. Elliott, X.T. Lu, K.W. Graepel, N.R. Sexton, E.C. Smith, S.R. Weiss, and M.R. Denison. 2017. Mouse hepatitis virus nsp14 exoribonuclease activity is required for resistance to innate immunity. Journal Virology 92 (1): e01531e01517. https://doi.org/10.1101/182196.

Chan, F.K., H.J. Chun, L. Zheng, R.M. Siegel, K.L. Bui, and M.J. Lenardo. 2000. A domain in TNF receptors that mediates ligand-independent receptor assembly and signaling. Science 288 (5475): 2351-2354. https://doi.org/10.112 6/science.288.5475.2351.

Chen, Y., H. Cai, J.A. Pan, N. Xiang, T.E. Po, T. Ahola, and D.Y. Guo. 2009. Functional screen reveals SARS coronavirus nonstructural protein nsp14 as a novel cap N7 methyltransferase. Proceedings of the National Academy of Sciences of the United States of America 106 (9): 3484-3489. https://doi.org/1 0.1073/pnas.0808790106.

Chen, Y., J.L. Tao, Y. Sun, A.D. Wu, C.Y. Su, G.Z. Gao, H. Cai, S. Qiu, Y.L. Wu, T. Ahola, et al. 2013. Structure-function analysis of severe acute respiratory syndrome coronavirus RNA cap guanine-N7-methyltransferase. Journal of Virology 87 (11): 6296-6305. https://doi.org/10.1128/JVI.00061-13.

de Artika, I.M., A.K. Dewantari, and A. Wiyatno. 2020. Molecular biology of coronaviruses: current knowledge. Heliyon 6 (8): e04743. https://doi.org/10.1 016/j.heliyon.2020.e04743.

Decroly, E., I. Imbert, B. Coutard, M. Bouvet, B. Selisko, K. Alvarez, A.E. Gorbalenya, E.J. Snijder, and B. Canard. 2008. Coronavirus nonstructural protein 16 is a cap-0 binding enzyme possessing (nucleoside-2'O)-methyltransferase activity. Journal of Virology 82 (16): 8071-8084. https://doi.org/10.1128/jvi.00407-08.

Gorbalenya, A.E., E.J. Snijder, and W.J. Spaan. 2004. Severe acute respiratory syndrome coronavirus phylogeny: toward consensus. Journal of Virology 78 (15): 7863-7866. https://doi.org/10.1128/jvi.78.15.7863-7866.2004. 
Haake, C., S. Cook, N. Pusterla, and B. Murphy. 2020. Coronavirus infections in companion animals: virology, epidemiology, clinical and pathologic features. Viruses 12 (9): 1023. https://doi.org/10.3390/v12091023.

Hayden, M.S., and S. Ghosh. 2008. Shared principles in NF-kB signaling. Cell 132 (3): 344-362. https://doi.org/10.1016/j.cell.2008.01.020.

Jin, X., Y. Chen, Y. Sun, C. Zeng, Y. Wang, J.L. Tao, A.D. Wu, X. Yu, Z. Zhang, J. Tian, et al. 2013. Characterization of the guanine-N7 methyltransferase activity of coronavirus nsp14 on nucleotide GTP. Virus Research 176 (1/2): 45-52. https:// doi.org/10.1016/j.virusres.2013.05.001.

Kanarek, N., and Y. Ben-Neriah. 2012. Regulation of NF-kB by ubiquitination and degradation of the IKBs. Immunological Reviews 246 (1): 77-94. https://doi. org/10.1111/j.1600-065x.2012.01098.x

Khatiwada, S., G. Delhon, P. Nagendraprabhu, S. Chaulagain, S.H. Luo, D.G. Diel, E. F. Flores, and D.L. Rock. 2017. A parapoxviral virion protein inhibits NF-KB signaling early in infection. PLoS Pathogens 13 (8): e1006561. https://doi.org/1 0.1371 /journal.ppat.1006561

Kumar, H., T. Kawai, and S. Akira. 2011. Pathogen recognition by the innate immune system. International Reviews of Immunology 30 (1): 16-34. https:// doi.org/10.3109/08830185.2010.529976.

Li, S.T., L.Y. Wang, and M.E. Dorf. 2009. PKC phosphorylation of TRAF 2 mediates IKKa/ $\beta$ recruitment and K63-linked polyubiquitination. Molecular Cell 33 (1): 30-42. https://doi.org/10.1016/j.molcel.2008.11.023.

Liu, F., Y.F. Xia, A.S. Parker, and I.M. Verma. 2012. IKK biology. Immunological Reviews 246 (1): 239-253. https://doi.org/10.1111/j.1600-065X.2012.01107.x.

Livak, K.J., and T.D. Schmittgen. 2001. Analysis of relative gene expression data using real-time quantitative PCR and the 2(-Delta Delta C (T)) method. Methods 25 (4): 402-408. https://doi.org/10.1006/meth.2001.1262.

Lu, Y.J., H. Cai, M.J. Lu, Y.M. Ma, A.Z. Li, Y.L. Gao, J.Y. Zhou, H. Gu, J.R. Li, and J.Y. Gu. 2020. Porcine epidemic diarrhea virus deficient in RNA cap guanine-N-7 methylation is attenuated and induces higher type I and III interferon responses. Journal of Virology 94 (16): e00447-e00420. https://doi.org/10.112 8/JVI.00447-20

Ma, Y.Y., L.J. Wu, N. Shaw, Y. Gao, J. Wang, Y.N. Sun, Z.Y. Lou, L.M. Yan, R.G. Zhang, and Z.H. Rao. 2015. Structural basis and functional analysis of the SARS coronavirus nsp14-nsp10 complex. Proceedings of the National Academy of Sciences of the United States of America 112 (30): 9436-9441. https://doi.org/1 0.1073/pnas.1508686112.

Minskaia, E., T. Hertzig, A.E. Gorbalenya, V. Campanacci, C. Cambillau, B. Canard, and J. Ziebuhr. 2006. Discovery of an RNA virus $3^{\prime}->5^{\prime}$ exoribonuclease that is critically involved in coronavirus RNA synthesis. Proceedings of the National Academy of Sciences of the United States of America 103 (13): 5108-5113. https://doi.org/10.1073/pnas.0508200103.

Nagendraprabhu, P., S. Khatiwada, S. Chaulagain, G. Delhon, and D.L. Rock. 2017. A parapoxviral virion protein targets the retinoblastoma protein to inhibit NF-kB signaling. PLoS Pathogens 13 (12): e1006779. https://doi.org/10.1371/ journal.ppat.1006779

O'Neill, L.A.J., and A.G. Bowie. 2010. Sensing and signaling in antiviral innate immunity. Current Biology 20 (7): R328-R333. https://doi.org/10.1016/j.cub.201 0.01.044.

Pires, B., R. Silva, G. Ferreira, and E. Abdelhay. 2018. NF-kappaB: two sides of the same coin. Genes 9 (1): 24. https://doi.org/10.3390/genes9010024

Poppe, M., S. Wittig, L. Jurida, M. Bartkuhn, J. Wilhelm, H. Müller, K. Beuerlein, N. Karl, S. Bhuju, J. Ziebuhr, M.L. Schmitz, and M. Kracht. 2017. The NF-kBdependent and -independent transcriptome and chromatin landscapes of human coronavirus 229E-infected cells. PLoS Pathogens 13 (3): e1006286. https://doi.org/10.1371/journal.ppat.1006286.

Rahman, M.M., and G. McFadden. 2011. Modulation of NF-KB signalling by microbial pathogens. Nature Reviews. Microbiology 9 (4): 291-306. https://doi. org/10.1038/nrmicro2539.

Rothwarf, D.M., E. Zandi, G. Natoli, and M. Karin. 1998. IKK- $\gamma$ is an essential regulatory subunit of the IKB kinase complex. Nature 395 (6699): 297-300. https://doi.org/10.1038/26261

Smits, S.L., A.N. de Lang, J.M.A. van den Brand, L.M. Leijten, W.F. van IJcken, M.J.C. Eijkemans, G. van Amerongen, T. Kuiken, A.C. Andeweg, A.D.M.E. Osterhaus, et al. 2010. Exacerbated innate host response to SARS-CoV in aged nonhuman Primates. PLoS Pathogens 6 (2): e1000756. https://doi.org/10.1371/ journal.ppat.1000756.

Sun, R.Q., R.J. Cai, Y.Q. Chen, P.S. Liang, D.K. Chen, and C.X. Song. 2012. Outbreak of porcine epidemic diarrhea in suckling piglets, China. Emerging Infectious Disease 18 (1): 161-163. https://doi.org/10.3201/eid1801.111259.
Tian, P.F., Y.L. Jin, G. Xing, L.L. Qv, Y.W. Huang, and J.Y. Zhou. 2014. Evidence of recombinant strains of porcine epidemic diarrhea virus, United States, 2013. Emerging Infectious Diseases 20 (10): 1735-1738. https://doi.org/10.3201/eid2 010.140338.

Wang, D., L.R. Fang, Y.L. Shi, H. Zhang, L. Gao, G.Q. Peng, H.C. Chen, K. Li, and S.B. Xiao. 2016. Porcine epidemic diarrhea virus 3C-like protease regulates its interferon antagonism by cleaving NEMO. Journal of Virology 90 (4): 2090 2101. https://doi.org/10.1128/JVl.02514-15.

Wang, K.Z., L.W. Ni, S. Wang, and C.F. Zheng. 2014. Herpes simplex virus 1 protein kinase $U_{3}$ hyperphosphorylates p65/RelA and dampens NF-kB activation. Journal of Virology 88 (14): 7941-7951. https://doi.org/10.1128/JVI.03394-13.

Woo, P.C.Y., S.K.P. Lau, C.S.F. Lam, C.C.Y. Lau, A.K.L. Tsang, J.H.N. Lau, R. Bai, J.L.L. Teng, C.C.C. Tsang, M. Wang, B.J. Zheng, K.H. Chan, and K.Y. Yuen. 2012. Discovery of seven novel Mammalian and avian coronaviruses in the genus Deltacoronavirus supports bat coronaviruses as the gene source of Alphacoronavirus and Betacoronavirus and avian coronaviruses as the gene source of Gammacoronavirus and Deltacoronavirus. Journal of Virology 86 (7): 3995-4008. https://doi.org/10.1128/JVI.06540-11.

Wullaert, A., M.C. Bonnet, and M. Pasparakis. 2011a. NF-kappaB in the regulation of epithelial homeostasis and inflammation. Cell Research 21 (1): 146-158. https://doi.org/10.1038/cr.2010.175.

Wullaert, Andy, Marion C. Bonnet, and Manolis Pasparakis. 2011b. NF-KB in the regulation of epithelial homeostasis and inflammation. Cell Research: 146158. https://doi.org/10.1038/cr.2010.175.

Xu, H.Y., C.H. Su, A. Pearson, C.H. Mody, and C.F. Zheng. 2017. Herpes simplex virus 1 UL24 abrogates the DNA sensing signal pathway by inhibiting NF-KB activation. Journal of Virology 91 (7): e00025-e00017. https://doi.org/10.1128/ jvi.00025-17.

Ye, R., C. Su, H. Xu, and C. Zheng. 2017. Herpes Simplex Virus 1 Ubiquitin-Specific Protease UL36 Abrogates NF-kappaB Activation in DNA Sensing Signal Pathway. Journal of Virology 91 (5): e02417-e02416. https://doi.org/10.1128/ JVI.02417-16

Zhang, J., K.Z. Wang, S. Wang, and C.F. Zheng. 2013a. Herpes simplex virus 1 E3 ubiquitin ligase ICP protein inhibits tumor necrosis factor alpha-induced NF$\mathrm{kB}$ activation by interacting with $\mathrm{p} 65 /$ RelA and p50/NF-kB1. Journal of Virology 87 (23): 12935-12948. https://doi.org/10.1128/JVI.01952-13.

Zhang, J., S. Wang, K.Z. Wang, and C.F. Zheng. 2013b. Herpes simplex virus 1 DNA polymerase processivity factor UL42 inhibits TNF-a-induced NF-KB activation by interacting with p65/RelA and p50/NF-kB1. Medicine Microbiology Immunology 202 (4): 313-325. https://doi.org/10.1007/s00430013-0295-0.

Zhang, Q.Z., H.Z. Ke, A. Blikslager, T. Fujita, and D. Yoo. 2017a. Type III interferon restriction by porcine epidemic diarrhea virus and the role of viral protein nsp1 in IRF, signaling. Journal of Virology 92 (4): JVI.01677-JVI.01617. https:// doi.org/10.1128/jvi.01677-17.

Zhang, Q.Z., J.Y. Ma, and D. Yoo. 2017b. Inhibition of NF-kB activity by the porcine epidemic diarrhea virus nonstructural protein 1 for innate immune evasion. Virology 510: 111-126. https://doi.org/10.1016/j.virol.2017.07.009.

Zhang, Q.Z., K.C. Shi, and D. Yoo. 2016. Suppression of type I interferon production by porcine epidemic diarrhea virus and degradation of CREBbinding protein by nsp1. Virology 489: 252-268. https://doi.org/10.1016/j. virol.2015.12.010

Zheng, D.H., G. Chen, B.C. Guo, G.H. Cheng, and H. Tang. 2008. PLP2, a potent deubiquitinase from murine hepatitis virus, strongly inhibits cellular type I interferon production. Cell Research 18 (11): 1105-1113. https://doi.org/10.103 8/cr.2008.294.

Ziebuhr, J., E.J. Snijder, and A.E. Gorbalenya. 2000. Virus-encoded proteinases and proteolytic processing in the Nidovirales. The Journal of General Virology 81 (Pt 4): 853-879. https://doi.org/10.1099/0022-1317-81-4-853.

\section{Publisher's Note}

Springer Nature remains neutral with regard to jurisdictional claims in published maps and institutional affiliations. 